\title{
Genome-wide association study suggests an independent genetic basis of zinc and cadmium concentrations in fresh sweet corn kernels
}

\author{
Matheus Baseggio (D) , ${ }^{1, \dagger}$ Matthew Murray, ${ }^{2}$ Di Wu (D) , ${ }^{1}$ Gregory Ziegler, ${ }^{3}$ Nicholas Kaczmar, ${ }^{1, \ddagger}$ James Chamness, ${ }^{1, \S}$ \\ John P. Hamilton (D) , ${ }^{\prime}$ C. Robin Buell (D) , ${ }^{4}$ Olena K. Vatamaniuk (D) , ${ }^{5}$ Edward S. Buckler (DD , 1,6,7 Margaret E. Smith, ${ }^{1}$ \\ Ivan Baxter (D) , William F. Tracy, ${ }^{3}$ and Michael A. Gore (iD ${ }^{1, *}$ \\ ${ }^{1}$ Plant Breeding and Genetics Section, School of Integrative Plant Science, Cornell University, Ithaca, NY 14853, USA \\ ${ }^{2}$ Department of Agronomy, University of Wisconsin-Madison, Madison, WI 53706, USA \\ ${ }^{3}$ Donald Danforth Plant Science Center, St. Louis, MO 63132, USA \\ ${ }^{4}$ Department of Plant Biology, Michigan State University, East Lansing, MI 48824, USA \\ ${ }^{5}$ Soil and Crop Sciences Section, Plant Biology Section, School of Integrative Plant Science, Cornell University, Ithaca, NY 14853, USA \\ ${ }^{6}$ Institute for Genomic Diversity, Cornell University, Ithaca, NY 14853, USA \\ ${ }^{7}$ US Department of Agriculture-Agricultural Research Service, Robert W. Holley Center for Agriculture and Health, NY 14853, USA \\ ${ }^{*}$ Corresponding author: Plant Breeding and Genetics Section, School of Integrative Plant Science, Cornell University, 358 Plant Science Building, Ithaca, NY 14853, \\ USA. Email: mag87@cornell.edu \\ ${ }^{\dagger}$ Present address: Seneca Foods Corporation, 1201 N. 4th Street, LeSueur, MN 56058, USA. \\ †Present address: Horticulture Section, School of Integrative Plant Science, Cornell University, Ithaca, NY 14853, USA. \\ Present address: Department of Genetics, Cell Biology, and Development, University of Minnesota, Saint Paul, MN 55108, USA.
}

\begin{abstract}
Despite being one of the most consumed vegetables in the United States, the elemental profile of sweet corn (Zea mays L.) is limited in its dietary contributions. To address this through genetic improvement, a genome-wide association study was conducted for the concentrations of 15 elements in fresh kernels of a sweet corn association panel. In concordance with mapping results from mature maize kernels, we detected a probable pleiotropic association of zinc and iron concentrations with nicotianamine synthase5 (nas5), which purportedly encodes an enzyme involved in synthesis of the metal chelator nicotianamine. In addition, a pervasive association signal was identified for cadmium concentration within a recombination suppressed region on chromosome 2 . The likely causal gene underlying this signal was heavy metal ATPase3 (hma3), whose counterpart in rice, OsHMA3, mediates vacuolar sequestration of cadmium and zinc in roots, whereby regulating zinc homeostasis and cadmium accumulation in grains. In our association panel, hma3 associated with cadmium but not zinc accumulation in fresh kernels. This finding implies that selection for low cadmium will not affect zinc levels in fresh kernels. Although less resolved association signals were detected for boron, nickel, and calcium, all 15 elements were shown to have moderate predictive abilities via whole-genome prediction. Collectively, these results help enhance our genomics-assisted breeding efforts centered on improving the elemental profile of fresh sweet corn kernels.
\end{abstract}

Keywords: genome-wide association study; whole-genome prediction; elements; kernels; sweet corn

\section{Introduction}

As with all multicellular organisms, the concentration and distribution of elements in tissues and organs influence growth and development over the plant life cycle. At least 16 elements (boron, calcium, carbon, chlorine, copper, hydrogen, iron, magnesium, manganese, molybdenum, nitrogen, oxygen, phosphorus, potassium, sulfur, and zinc) are considered essential for higher plant species, with an additional four elements (cobalt, silicon, nickel, and sodium) essential for a subset of higher plants (Mengel and Kirkby 2001). In plants, the need for and concentration of macroelements (carbon, hydrogen, oxygen, nitrogen, phosphorus, sulfur, potassium, calcium, magnesium) (Hawkesford et al. 2012) are relatively greater than for microelements (iron, manganese, copper, zinc, nickel, molybdenum, boron, chlorine, and cobalt) (Broadley et al. 2012). In addition, nonessential heavy metals such as cadmium, chromium, and lead that lack involvement in normal physiological functions can accumulate to toxic concentrations in plants, penetrating the food chain and posing a threat to human health (Singh et al. 2016).

Not unlike plants, humans can suffer a range of adverse health effects from the excess or deficiency of essential and nonessential elements. Of all the micronutrients, deficiency is most prevalent for iron, with more than two billion people affected worldwide (Viteri 1998). Of similar scale, nearly two billion people 
are estimated to suffer from dietary zinc deficiency throughout developing nations (Prasad 2014). Given that metal chelating substances such as phytate in cereal grains bind zinc and iron and inhibit their absorption, zinc and iron deficiencies resulting from low bioavailability could coincide (Sandstead and Smith 1996; Lönnerdal 2000). Although severe dietary micronutrient deficiency is far less prevalent in developed nations, approximately 10 million people are iron deficient in the U.S. (Miller 2013). In the U.S., the average daily intake of iron by most premenopausal $\left(12 \mathrm{mg} \mathrm{d}^{-1}\right)$ and pregnant $\left(15 \mathrm{mg} \mathrm{d}^{-1}\right)$ women is 6 and $12 \mathrm{mg} \mathrm{d}^{-1}$ less than its recommended daily allowance (RDA), respectively (Institute of Medicine 2001; Linus Pauling Institute 2016). In addition, the zinc RDA for adult women and men is $11 \mathrm{mg} \mathrm{d}^{-1}$ and $8 \mathrm{mg} \mathrm{d}^{-1}$, with elderly at higher risk for mild zinc deficiency (Mocchegiani et al. 2013; Linus Pauling Institute 2019).

Crop biofortification via genomics-assisted breeding and genetic engineering has emerged as an attractive approach for nutritional enhancement of crops and the generation of new varieties with a high density of iron and zinc in edible plant tissues (Murgia et al. 2012; Bhullar and Gruissem 2013; Hirschi 2020). It is increasingly recognized that plant membrane transporters and metal chelators are among the key targets for increasing mineral nutrient density in plant tissues (Waters and Sankaran 2011; Schroeder et al. 2013). However, in many cases, transporters that facilitate the accumulation of iron and zinc are multispecific and can mediate the uptake and internal transport of nonessential and potentially highly toxic heavy metals including cadmium (Waters and Sankaran 2011; Schroeder et al. 2013; Khan et al. 2014). Therefore, efforts to increase the concentration of iron and zinc in grains of cereals could also increase the concentration of cadmium. This poses a serious threat to food security, especially if crops are grown on soils either contaminated with cadmium or low in microelements, particularly iron (Waters and Sankaran 2011; Schroeder et al. 2013; Khan et al. 2014).

Vegetative and seed tissues of fruits and vegetables are important dietary sources of essential and nonessential elements for humans to meet their daily nutrient needs. Given that sweet corn is the third most consumed vegetable in the U.S. (USDA-NASS 2018), the elemental profile of fresh sweet corn kernels is an important consideration for human health and nutrition. Although not a major contribution to the RDA of iron and zinc, the consumption of $100 \mathrm{~g}$ of uncooked, yellow sweet corn (medium-sized ear) provides $0.52 \mathrm{mg}$ of iron and $0.46 \mathrm{mg}$ of zinc (USDA-ARS 2019), but the bioavailable amount is expected to be no more than a quarter of the total of each element (Bouis and Welch 2010). Therefore, there exists a tremendous opportunity to improve the elemental profile of fresh sweet corn kernels through genomics-assisted breeding, but this first requires an understanding of the phenotypic variability and genetic control of zinc, iron, and other elements. Considerable heritable variation exists for elemental concentrations in physiologically mature grain of diverse maize panels (Ziegler et al. 2017; Wu et al. 2021), but a comparable level of genetic understanding is severely lacking for immature kernels (fresh-eating stage) of diverse sweet corn germplasm.

Complex physiological and genetic networks coordinate elemental uptake, transport, and accumulation in plants, and these processes are responsive to the environment in which plants are grown (Reviewed in Baxter 2009). In the genomes of Arabidopsis, maize, rice, and other plant species, gene families have been identified for metal transporters and chelators including but not limited to HEAVY-METAL ATPASE (HMA), OLIGOPEPTIDE TRANSPORTERS (OPTS), and their subfamily of YELLOW STRIPE-
LIKE (YSL), ZINC-REGULATED TRANSPORTER (ZRT)/IRONREGULATED TRANSPORTER (IRT)-LIKE PROTEIN (ZIP), and NICOTIANAMINE SYNTHASE (NAS) (Whitt et al. 2020). As it relates to maize, yellow stripe1 (ys1) and ys3 encode proteins that have been functionally shown to transport iron when associated with the strong metal-ligand, nicotianamine-synthesized from S-adenosyl-methionine by NAS enzymes-or its derivative phytosiderophores such as mugineic acid and deoxymugenic acid (Von Wiren et al. 1994; Chan-Rodriguez and Walker 2018), whereas the proteins encoded by ysl2 and zip5 have been functionally implicated in the accumulation of zinc and iron in grain (Li et al. 2019; Zang et al. 2020). Despite these advancements, the vast majority of metal transporters and chelators in the maize genome have not been deeply characterized at the functional level, thus a wide gap in the knowledge base remains for the key gene family members controlling the content and composition of elements in maize tissues and organs.

Explaining and predicting the quantitative variation of phenotypes is a major challenge in crop plants, but there has been notable recent progress for maize grain elemental phenotypes (Ziegler et al. 2017; Hindu et al. 2018; Wu et al. 2021). In the U.S. maize nested association mapping (NAM) panel, joint-linkage analysis and genome-wide association studies (GWAS) were used to identify six strong candidate genes for the concentrations of manganese, molybdenum, phosphorus, or rubidium in physiologically mature grain (Ziegler et al. 2017). Through the implementation of GWAS in the maize Ames panel, Wu et al. (2021) resolved several loci previously identified to control variation for copper, iron, manganese, molybdenum, and/or zinc in mature grain from the U.S. NAM panel, which resulted in the identification of two metal chelator and five metal transporter candidate genes. In addition, the authors detected novel candidate gene loci for boron and nickel grain concentrations. Whole-genome prediction (WGP) models have been found to be moderately predictive of elemental concentrations in mature maize grain of tropical populations (zinc) (Guo et al. 2020; Mageto et al. 2020) and the Ames panel (boron, calcium, copper, iron, potassium, magnesium, manganese, molybdenum, nickel, phosphorus, and zinc) (Wu et al. 2021). Notwithstanding this progress with mature grain, the genotype-phenotype map of elemental concentrations in fresh sweet corn kernels is completely nonexistent, thus there exists tremendous opportunities for studying the quantitative genetics of these nutritionally relevant phenotypes.

In this study, we used a sweet corn association panel for the genetic dissection and prediction of quantitative variation of 15 elements in fresh sweet corn kernels. The three major objectives of our study were to (i) evaluate variability and heritability of elemental fresh kernel phenotypes within and across field locations, (ii) employ GWAS to identify candidate genes associated with the levels of elements in fresh kernels, and (iii) assess the predictive abilities of WGP models as an evaluation of the potential that genomic selection has for the genetic improvement of elemental fresh kernel phenotypes important to human nutrition and health.

\section{Materials and methods Plant materials and experimental design}

In two consecutive field seasons (2014 and 2015), a sweet corn association panel of 430 inbred lines representing the genetic diversity of temperate U.S. breeding programs (Baseggio et al. 2019) was evaluated at Cornell University's Musgrave Research Farm in Aurora, NY, on a Lima silt loam (fine-loamy, mixed, semiactive, 
mesic Oxyaquic Hapludalfs) and University of Wisconsin's West Madison Research Station in Verona, WI, on a Plano silt loam (fine-silty, mixed, superactive, mesic Typic Argiudolls). The panel consists of sugary1 (su1), sugary1: sugary enhancer1 (su1se1), shrunken2 (sh2), sugary1: shrunken2 (su1sh2), brittle2 (bt2), and amylose-extender: dull: waxy (aeduwx) lines that are homozygous for endosperm mutations that cause deficiencies in starch biosynthesis. In addition, there were 20 nonsweet corn inbred lines and four repeated check sweet corn inbred lines included in the experiment. In each of the four environments (location $\times$ year combination), the experiment was arranged as an augmented incomplete block design grown as a single replicate as previously described by Baseggio et al. (2019). Briefly, the lines were separated into three sets according to their plant height, with each set having incomplete blocks. Each incomplete block of 20 experimental lines was augmented with the random placement of two height-specific check lines (We05407 and W5579, W5579 and Ia5125, or Ia5125 and IL125b). In both field locations, experimental units were one-row plots of different lengths. Plots were $3.05 \mathrm{~m}$ long in NY and $3.50 \mathrm{~m}$ long in WI. Both locations had an inter-row spacing of $0.76 \mathrm{~m}$, with a $0.91 \mathrm{~m}$ alley at the end of each plot. In NY, 25 kernels were planted per plot and thinned to 12 plants per plot. In WI, 12 kernels were planted in each plot, but plots were not thinned.

In all environments, multiple plants per plot were selfed pollinated, with two selfed ears collected by hand from each harvestable plot at 400 growing degree days after pollination (i.e., immature kernel stage at approximately 21 days after pollination) as earlier described (Baseggio et al. 2019). Immediately upon fresh harvest, the entirety of each dehusked ear was directly frozen in liquid nitrogen, followed by hand shelling of frozen kernels. To generate a representative composite kernel sample for each harvested plot, frozen kernels were equally sampled at random from both ears, bulked, and stored in $15 \mathrm{ml}$ Falcon tubes at $-80^{\circ} \mathrm{C}$ until lyophilization. A combined set of 1524 plot samples from across all environments, with each sample consisting of three lyophilized kernels, was shipped to the Donald Danforth Plant Science Center (St. Louis, MO, USA) for elemental analysis.

\section{Phenotypic data analysis}

For each plot sample, the determination of elemental concentration by an inductively coupled plasma mass spectrometer (ICP-MS) was conducted separately for each of the three lyophilized kernels as previously described in Baxter et al. (2014). In short, each individual unground kernel was robotically weighed, digested in concentrated nitric acid, and measured for concentrations of aluminum, arsenic, boron, cadmium, calcium, cobalt, copper, iron, magnesium, manganese, molybdenum, nickel, phosphorus, potassium, rubidium, selenium, sodium, strontium, sulfur, and zinc with a PerkinElmer NexION 350 D ICP-MS. Of these 20 elements, aluminum, arsenic, cobalt, selenium, and sodium were not further considered because their measured concentrations were at trace levels, vulnerable to contamination in the course of sample processing, and/or sensitive to interference from other sample matrix constituents (Ziegler et al. 2013). To limit the influence of extreme analytical outliers that could negatively affect the accurate estimation of variance components when initially fitting a mixed linear model to the raw data, the method of Davies and Gather (1993) was implemented similarly to its use in Baxter et al. (2014) to remove raw concentration values with greater than a conservative threshold of 15 median absolute deviations from the median concentration for a given element within each environment. Also, if less than $1 \%$ of the values for a given element were negative, these negative values were set to missing.

The preliminarily processed raw ICP-MS dataset was more robustly screened for significant outliers by fitting a mixed linear model that allowed for genetic effects to be separately estimated from field design effects, following the procedure described in Wolfinger et al. (1997). The fitted mixed linear model was similar to that used by Baseggio et al. (2019) for the same experimental field design, with the notable exception that the model used in this study included a term to estimate within-plot kernel sample variance. This allowed for the removal of individual outlier measurements. For each elemental phenotype, the full model was fitted in ASReml-R version 3.0 (Gilmour et al. 2009) across locations (all four environments) or for each location separately (two environments, NY; or two environments, WI) as follows:

$$
\begin{aligned}
\mathrm{Y}_{\mathrm{ijklmnopq}}= & \mu+\text { check }_{i}+\text { env }_{j}+\operatorname{set}(e n v)_{j k}+\text { block }(\text { set } \times e n v)_{j k l} \\
& + \text { genotype }_{m}+(\text { genotype } \times \text { env })_{j m}+\text { ICP-MS.run } n \\
& + \text { sample }_{o}+\text { row }(e n v)_{j p}+\operatorname{col}(\text { env })_{j q}+\varepsilon_{i j k l m n o p q}
\end{aligned}
$$

in which $Y_{\text {ijklmnopq }}$ is an individual phenotypic observation, $\mu$ is the grand mean, check $k_{i}$ is the fixed effect for the ith check, env $j$ is the effect of the $j$ th environment, set(env $)_{j k}$ is the effect of the kth set within the jth environment, block(set $\times$ env) jkl is the effect of the lth incomplete block within the kth set within the jth environment, genotype $m$ is the effect of the $m$ th experimental genotype (noncheck line), (genotype $\times$ env) jm is the effect of the interaction between the $m$ th genotype and jth environment, ICP-MS.run $n_{n}$ is the laboratory effect of the $n$th ICP-MS run, sample is the oth kernel sample, row(env) $)_{j p}$ is the effect of the pth plot grid row within the jth environment, col(env $)_{j q}$ is the effect of the qth plot grid column within the jth environment, and $\varepsilon_{i j k l m n o p q}$ is the heterogeneous residual error effect within each environment with a firstorder autoregressive correlation structure among plot residuals in the row and column directions. Except for the grand mean and check term, all terms were modeled as random effects. The Kenward-Roger approximation (Kenward and Roger 1997) was used to calculate degrees of freedom. Studentized deleted residuals (Neter et al. 1996) obtained from these mixed linear models were used to detect significant outliers for each phenotype after a Bonferroni correction $(\alpha=0.05)$.

To generate the best linear unbiased predictor (BLUP) values for each elemental phenotype, an iterative mixed linear model fitting procedure was conducted on the outlier-screened phenotypic dataset in ASReml-R version 3.0 (Gilmour et al. 2009) with the full model across locations or for each location separately. Model terms fitted as random effects including the autoregressive correlations were tested with likelihood ratio tests (Littell et al. 2006), followed by the removal of terms from the model that were not significant at $\alpha=0.05$. The significance of main random effects and variance component estimates are reported in Supplementary Table S1. In addition, the first-order autoregressive correlation structure was statistically significant for all phenotypes. For each elemental phenotype, the final, best-fitted model was used to generate a BLUP for each inbred line. The generated BLUP values were filtered to remove nonsweet corn lines, as well as sweet corn lines with the infrequent aeduwx or bt2 endosperm mutations and those without available SNP marker data. This resulted in 401 sweet corn lines with more prevalent endosperm mutations [su1, su1se1 (classified as su1 for this study due to lack of informative marker genotypes), sh2, and su1sh2] 
that had BLUP values for elemental phenotypes across and within locations.

With variance component estimates from each best fitted model, heritability on a line-mean basis was calculated for each elemental phenotype across locations and separately for each location as previously described (Lynch and Walsh 1998; Holland et al. 2003; Hung et al. 2012). Pearson's correlation coefficient ( $r$ ) was used to assess the degree of association between the BLUP values of paired phenotypes. Pairwise correlations were calculated, and their significance tested at $\alpha=0.05$ with the method "pearson" from the function "cor.test" in R version 3.6.1 (R Core Team 2019).

\section{SNP marker genotyping}

The sweet corn inbred association panel was sequenced via the genotyping-by-sequencing (GBS) procedure of Elshire et al. (2011) with ApeKI at the Cornell Biotechnology Resource Center (Cornell University, Ithaca, NY, USA) as previously described (Baseggio et al. 2019). The procedure of Baseggio et al. (2020) for SNP calling, filtering, and imputing missing genotypes was used to construct a SNP marker dataset for the genetic dissection and prediction of fresh kernel elemental phenotypes. In brief, the raw GBS sequencing data from Baseggio et al. (2019) was processed through the production pipeline in TASSEL 5 GBSv1 with the ZeaGBSv2.7 Production TagsOnPhysicalMap file to call SNPs at 955,690 loci in B73 RefGen_v2 coordinates (Glaubitz et al. 2014). These raw SNP genotype calls were merged with those of 19 sweet corn inbred lines from Romay et al. (2013) that were not included in the Baseggio et al. (2019) GBS dataset, allowing for the assemblage of raw SNP calls for all 401 sweet corn inbred lines with BLUP values.

The combined raw dataset was initially filtered by keeping only biallelic SNPs with a call rate $>10 \%$ and eliminating singleton (heterozygous site) and doubleton (homozygous site) SNPs that scored a minor allele in only a single individual. Given the potential to have resulted from paralogous alignments, we set heterozygous genotype calls with an allele balance score (lowest allele read depth/total read depth) $<0.3$ to missing. If multiple GBS samples existed for an inbred line, SNP genotype calls from samples with the same accession number/identifier were merged and discordant genotype calls set to missing if identical-by-state (IBS) values for all within-line sample comparisons were $>0.99$, following the conservative IBS threshold set by Romay et al. (2013). A single GBS sample with the highest SNP call rate was chosen to represent an inbred line if all pairwise IBS values were less than 0.99 .

The FILLIN haplotype-based imputation strategy of Swarts et al. (2014) was used to impute missing SNP genotypes to near completeness based on a set of maize haplotype donors with a window size of $4 \mathrm{~kb}$. To improve the quality of the imputed dataset, we filtered SNPs in TASSEL 5 version 20190321 to remove those with a call rate $<70 \%$ (residual missing genotype data are expected for the haplotype-based imputation method of FILLIN; Swarts et al., 2014), a minor allele frequency $<5 \%$, heterozygosity $>10 \%$, coefficient of panmixia $<80 \%$, or a mean read depth $>15$. To uplift the genome coordinates of retained SNPs to B73 v4, the Vmatch software (Kurtz 2003) was used to align the 101 bp context sequence of each SNP to the B73 RefGen_v4 reference genome, resulting in 147,762 high-quality SNP markers scored on the 401 sweet corn inbred lines.

\section{Genome-wide association study}

A GWAS was conducted across and within locations to identify SNP markers significantly associated with each elemental phenotype following the methods of Baseggio et al. (2020) with minor modifications. In short, the Box-Cox power transformation (Box and Cox 1964) was used with an intercept-only model to choose the optimal value of convenient lambda $(-2$ to $+2,0.5$ increments) (Supplementary Table S2) for transforming the BLUP values of each elemental phenotype to lessen heteroscedasticity and nonnormality of the residuals with the MASS package in R version 3.6.1 (R Core Team 2019). For each elemental phenotype, a mixed linear model (Yu et al. 2006; Zhang et al. 2010) that accounted for population structure and unequal relatedness with principal components (PCs) and a genomic relationship matrix (GRM; kinship) was used to test for an association between each of the 147,762 SNPs and transformed BLUP values in GEMMA software version 0.97 (Zhou and Stephens 2014). In the R package GAPIT version 2017.08.18 (Lipka et al. 2012), 10,773 unimputed genome-wide SNPs (call rate $>90 \%$, MAF $>5 \%$, heterozygosity $<10 \%$, coefficient of panmixia $>80 \%$, and mean read depth $<15$ ) subsampled from the complete marker dataset were used to calculate PCs with the prcomp function and the kinship matrix with VanRaden's method 1 (VanRaden 2008). The conservative imputation of residual missing SNP genotypes as heterozygous in both marker datasets was conducted in GAPIT.

The Bayesian information criterion (BIC) (Schwarz 1978) was used to ascertain the optimal number of PCs to incorporate in the mixed linear model. Given that the predominant accumulation of some elements in the endosperm (Lombi et al. 2009, 2011; Pongrac et al. 2013; Baxter et al. 2014; Cheah et al. 2019) could potentially lead to likely spurious associations with su1 and sh2 as shown for tocotrienols and certain carotenoids (Baseggio et al. 2019, 2020), endosperm mutation type (su1, sh2, or su1sh2) was also tested with the BIC for inclusion as a covariate in the model. Of the 401 inbred lines, 384 lines had endosperm mutation type scores available from Baseggio et al. (2019), whereas endosperm mutation type for each of the remaining 17 lines without visual scores was predicted with the identical optimal marker-based classification models and $1000 \mathrm{~kb}$ marker datasets for the su1 and sh2 loci from Baseggio et al. (2019).

To approximate the amount of phenotypic variation explained by a significantly associated SNP, we calculated the difference between the likelihood-ratio-based $R^{2}$ statistic $\left(R_{L R}^{2}\right)$ of Sun et al. (2010) from a mixed linear model with or without the given SNP, following Baseggio et al. (2020). The false discovery rate (FDR) was controlled at $5 \%$ by adjusting the P-values (Wald test) of SNPs tested in GEMMA using the Benjamini-Hochberg multiple test correction (Benjamini and Hochberg 1995) with the "p.adjust" function in $\mathrm{R}$ version 3.6.1 ( $\mathrm{R}$ Core Team 2019). Given the large variance in the estimated distance to which median genomewide linkage disequilibrium (LD) decays to background levels $\left(r^{2}<0.1\right.$ by $\sim 12 \mathrm{~kb}$ ) in this association panel (Baseggio et al. 2019) and to account for the possibility of distant cis-regulatory elements (Ricci et al. 2019), candidate gene searches were limited to $\pm 250 \mathrm{~kb}$ (median $r^{2} \leq 0.05$ ) of the physical position of SNP markers significantly associated with an elemental phenotype. For each most plausible candidate gene, we used BLASTP to identify the top three unique best hits (E-values $<1$ ) in Arabidopsis (Columbia-0 ecotype) and rice (Oryza sativa L. ssp. Japonica cv. "Nipponbare") using default parameters at the TAIR (https:// www.arabidopsis.org) and RAP-DB (https://rapdb.dna.affrc.go.jp) databases, respectively. The across-location (All Locs: New York, 
Florida, North Carolina, and Puerto Rico) results from jointlinkage analysis and GWAS of grain elemental phenotypes in the U.S. NAM panel (Ziegler et al. 2017) were integrated with the physical (bp) positions of GWAS signals from our study in B73 RefGen_AGPv4 coordinates following the approach of Wu et al. (2021).

The multi-locus mixed-model (MLMM) approach of Segura et al. (2012) that sequentially adds significant markers as covariates in the model was used to better clarify significant association signals with underlying large-effect loci at the level of an individual chromosome as previously described (Lipka et al. 2013). The optimal model was selected with the extended BIC (Chen and Chen 2008). To further assess the extent of statistical control for large-effect loci, GWAS was reconducted by including MLMM-selected SNPs as fixed effects (covariates) in mixed linear models fitted in GEMMA.

\section{Linkage disequilibrium}

The local patterns of LD surrounding significantly associated loci were investigated by estimating pairwise LD between SNPs with the squared allele-frequency correlation $\left(r^{2}\right)$ method of Hill and Weir (1988) in TASSEL 5 version 20190627 (Bradbury et al. 2007). The marker dataset used for estimation of LD consisted of the 147,762 SNPs without imputation of the post-FILLIN residual missing SNP genotypes to heterozygotes.

\section{Whole-genome prediction}

A univariate genomic best linear unbiased prediction (GBLUP) model (Bradbury et al. 2007; VanRaden 2008) was used to evaluate WGP on the transformed across-location BLUP values of the 15 elemental phenotypes as previously described by Baseggio et al. (2020). In short, the 401 line $x$ 147,762 SNP genotype matrix with post-FILLIN missing data imputed as a heterozygous genotype was used to construct a GRM with method 1 from VanRaden (2008) in GAPIT version 2017.08.18 (Lipka et al. 2012). Next, the constructed GRM was modeled as a random effect to predict each individual elemental phenotype with the function "emmreml" in version 3.1 of the R package EMMREML (Akdemir and Okeke 2015). Through the implementation of a fivefold cross-validation scheme conducted 50 times for each elemental phenotype, the predictive ability of a phenotype was calculated as the mean Pearson's correlation between transformed BLUP (observed) and genomic estimated breeding values (predicted). Each fold was representative of genotype frequencies for endosperm mutants (su1, sh2, and su1sh2) observed in the whole association population. Endosperm mutation type (su1, sh2, or su1sh2) was also evaluated as a covariate in prediction models, with the same cross-validation folds used across models with or without the covariate for endosperm mutation type.

\section{Data availability}

All raw GBS sequencing data are available from the National Center of Biotechnology Information Sequence Read Archive under accession number SRP154923 and in BioProject under accession PRJNA482446. The ZeaGBSv2.7 Production TagsOnPhysicalMap file (AllZeaGBSv2.7_ProdTOPM_20130605.topm.h5) for calling SNPs, the raw SNP genotype data in B73 AGPv2 coordinates (ZeaGBSv27_publicSamples_rawGenos_AGPv2-150114.h5) for the 19 sweet corn lines of Romay et al. (2013), and the maize haplotype donor file (AllZeaGBSv2.7impV5_AnonDonors4k.tar.gz) for imputing missing genotypes are on CyVerse (https://datacommons. cyverse.org/browse/iplant/home/shared/panzea/genotypes/GBS/ v27). The BLUP values of the 15 elemental phenotypes and the
FILLIN imputed SNP genotype calls in B73 AGPv4 coordinates for the 401 inbred lines are available at CyVerse: (https://data commons.cyverse.org/browse/iplant/home/shared/GoreLab/ dataFromPubs/Baseggio_SweetcornElement_2021). All supplementary materials are available at figshare: https://doi.org/10. 25387/g3.14633028. Except for the University of Wisconsin germplasm, all inbred lines included in the sweet corn association panel are in the public domain. A material transfer agreement is required to obtain some of the Wisconsin lines.

\section{Results \\ Phenotypic variation}

The extent of phenotypic variation for 15 elements in fresh kernels as quantified by ICP-MS was evaluated in an association panel of 401 sweet corn inbred lines that was grown in two field locations (Verona, WI; and Aurora, NY) in 2014 and 2015. Of the five macroelements studied, potassium, phosphorus, sulfur, and magnesium had average concentrations greater than $1000 \mu \mathrm{g} \mathrm{g}^{-1}$, whereas calcium had an average concentration of nearly $60 \mu \mathrm{g}$ $\mathrm{g}^{-1}$ (Table 1). Average concentrations ranged from 0.012 (cadmium) to 24.52 (zinc) $\mu \mathrm{g} \mathrm{g}^{-1}$ for the 10 microelements. Even though cadmium had the lowest mean concentration, it had a 12.11-fold range in variation (maximum BLUP value divided by the minimum BLUP value), whereas the other 14 elements covered a 1.34- to 3.66-fold range in variation. When separating inbred lines according to their endosperm mutation type (Table 2), copper, iron, manganese, and potassium were found to be at significantly $(P<0.001)$ greater concentrations in the sh2 $(n=78)$ group relative to the su1 $(n=301)$ group.

Implying common genetic control (Baxter 2015), shared chemical and physiological properties (Marschner 2011), or storage with phytic acid (Maathuis 2009), element pairs with strong positive correlations $(r>0.50 ; \quad P<0.01)$ across locations (Supplementary Figure S1) were as follows: strontium/calcium, magnesium/phosphorus, zinc/iron, and phosphorus/zinc. Suggestive of a distinct genetic architecture, molybdenum was the element most weakly correlated with other elements across locations (Supplementary Figure S1), having a significant but

Table 1 Means and ranges for untransformed best linear unbiased predictors (BLUPs) of 15 fresh kernel elemental phenotypes evaluated in the sweet corn association panel and estimated heritability $\left(\hat{h}_{l}\right)$ on a line-mean basis across two years and two locations

\begin{tabular}{|c|c|c|c|c|c|}
\hline \multirow[b]{2}{*}{ Trait } & \multirow[b]{2}{*}{ Lines } & \multicolumn{3}{|c|}{ BLUPs } & \multirow[b]{2}{*}{$\hbar_{l}^{2}$} \\
\hline & & Mean & $\mathrm{SD}^{\mathrm{a}}$ & Range & \\
\hline & & \multicolumn{3}{|c|}{ _ $\mu$ g g-1 dry weight } & \\
\hline Boron & 399 & 1.89 & 0.12 & $1.62-2.35$ & 0.15 \\
\hline Cadmium & 401 & 0.012 & 0.006 & 0.003 & 0.83 \\
\hline Calcium & 392 & 57.91 & 10.76 & 34.36 & 0.48 \\
\hline Copper & 401 & 3.25 & 0.76 & 1.61 & 0.81 \\
\hline Iron & 401 & 18.55 & 2.08 & $13.66-28.11$ & 0.64 \\
\hline Magnesium & 401 & 1268.23 & 71.83 & $1,088.97-1,574.82$ & 0.64 \\
\hline Manganese & 401 & 8.52 & 1.21 & $5.33-13.13$ & 0.86 \\
\hline Molybdenum & 400 & 301 & 0.040 & 0.209 & 0.88 \\
\hline Nickel & 401 & 0161 & 0.032 & 0.096 & 0.64 \\
\hline Phosphorus & 401 & $3,186.06$ & 165.14 & $2,713.77-3,639.37$ & 0.76 \\
\hline Potassium & 401 & $8,089.79$ & 473.62 & $6,558.37-9,765.09$ & 0.76 \\
\hline Rubidium & 401 & 2.72 & 0.25 & $2.22-3.72$ & 0.58 \\
\hline Strontium & 401 & 0.273 & 0.044 & $0.177-0.503$ & 0.60 \\
\hline Sulfur & 401 & $1,451.63$ & 73.60 & $1,258.22-1,712.46$ & 0.40 \\
\hline Zinc & 401 & 24.52 & 2.26 & $18.58-32.10$ & 0.75 \\
\hline
\end{tabular}

${ }^{\text {a }}$ Standard deviation of the BLUPS. 
Table 2 Estimated effects of endosperm mutation type from untransformed best linear unbiased predictors of 15 fresh kernel elemental phenotypes across 2 years and two locations

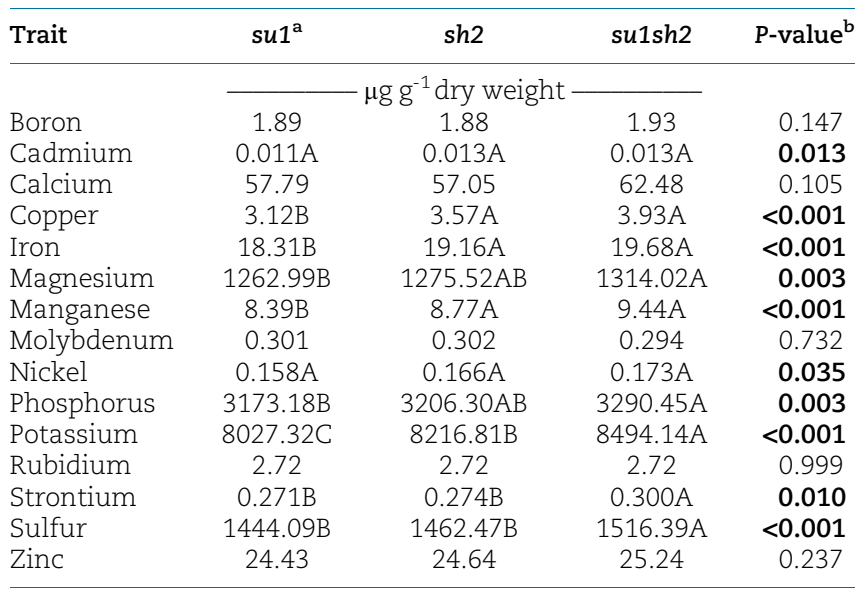

a Sweet corn lines grouped by endosperm mutation type having labels with the same letter are not significantly different according to the Tukey-Kramer honest significant difference test $(P<0.05)$. The test was only performed for traits that had a significant F-test.

b P-value from one-way analysis of variance (ANOVA) F-test for the endosperm mutation type effect. Bolded P-value indicates a statistically significant difference between two or more endosperm mutation type groups $(P<0.05)$.

very weak positive correlation $(r=0.12 ; P=0.02)$ with zinc alone. Apart from boron $\left(\hat{h}_{l}^{2}=0.15\right)$ that can have elevated background levels from the use of glass (sodium borosilicate) tubes for chemical digestion (Baxter et al. 2014), the across-location heritability estimates (Table 1) for the elemental phenotypes were 0.40 (sulfur) and larger. The $\mathrm{G} \times \mathrm{E}$ interaction term was only significant for boron, copper, iron, magnesium, and rubidium, whereas the genotype term was significant for all 15 elements (Supplementary Figure S2 and Table S1).

When investigating phenotypic variation between locations, we found that only the average concentration of sulfur was not significantly different between locations (Supplementary Figure S3). Indicative of phenotypes with a range of responsiveness to the environment, the correlation ( $r$ ) of elemental trait BLUPs between locations ranged from 0.08 (boron) to 0.68 (copper), with an average correlation of 0.42 (Supplementary Figure S4). Despite the mostly moderate correlations between locations, within-location heritability estimates (Supplementary Table S3) were comparable to those estimated across locations (Table 1) and strongly correlated $(r=0.81)$ between the NY and WI locations. Altogether, our findings suggest that there is value in exploring the genetic dissection of elemental fresh kernel phenotypes across- and within-locations.

\section{Genome-wide association study}

We investigated the genetic basis of natural variation for the concentration of 15 elements in fresh kernels from the sweet corn association panel of 401 inbred lines that had been evaluated in four environments (2 years $\times$ two locations) and scored with 147,762 genome-wide SNP markers. Through an across-location GWAS conducted with a unified mixed linear model that accounted for population structure, relatedness, and endosperm mutation type, 220 unique SNPs were found to associate with one of three elements (cadmium, zinc, or boron) at a genome-wide FDR of 5\% (Supplementary Table S4). Significant association signals were only found on chromosomes 1 (boron), 2 (cadmium), and 7 (zinc), with the exception of a single SNP associated with cadmium on chromosome 8 (Supplementary Figure S5).

The strongest association signal was identified for the concentration of cadmium, consisting of 191 significant SNPs that covered a 36.03-Mb interval within a long-range $\mathrm{LD}$ region of chromosome 2 (Figure 1A). The peak SNP (S2_157751802; P-value $\left.1.53 \times-10^{23} ; 162,398,589 \mathrm{bp}\right)$ for this complex association signal (Supplementary Table S4), which explained $18 \%$ of the phenotypic variance for cadmium, was positioned within the open reading frame (ORF) of a gene (Zm00001d005174) that codes for a protein that belongs to the superfamily of uridine diphosphateglycosyltransferases (www.maizegdb.org). However, this and other candidate genes within $250 \mathrm{~kb}$ of the peak SNP (www.mai zegdb.org) were considered to unlikely be involved in cadmium accumulation. Given the extensive LD within this recombination suppressed region (Gore et al. 2009; Rodgers-Melnick et al. 2016),
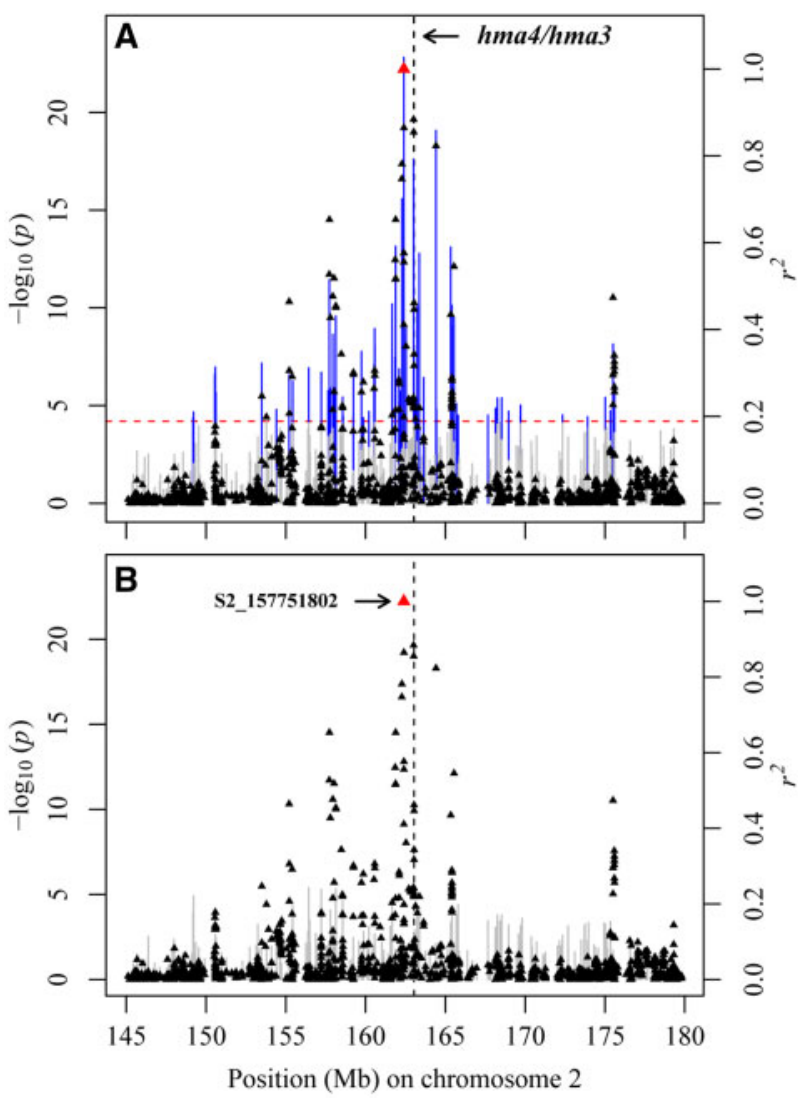

Figure 1 Genome-wide association study for cadmium concentration in fresh kernels of sweet corn. (A) Scatter plot of association results from a mixed model analysis and linkage disequilibrium (LD) estimates $\left(r^{2}\right)$. The vertical lines are - $\log _{10} P$-values of single nucleotide polymorphisms (SNPs), with the blue color representing SNPs that are statistically significant at a 5\% false discovery rate (FDR). Triangles are the $r^{2}$ values of each SNP relative to the peak SNP (indicated in red) at 162,398,589 bp (B73 RefGen_v4) on chromosome 2. The red horizontal dashed line indicates the $-\log _{10} P$-value of the least statistically significant SNP at a $5 \%$ FDR. The black vertical dashed lines indicate the genomic positions of the heavy metal ATPase4 (hma4; Zm00001d005189; 163,016,710163,020,248 bp) and heauy metal ATPase3 (hma3; Zm00001d005190; $163,038,225-163,041,426 \mathrm{bp}$ ) genes. These two genes are separated by a physical distance of $\sim 18 \mathrm{~kb}$, thus their positions are not distinguishable at the plotted scale. (B) Scatter plot of association results from a conditional mixed linear model analysis and LD estimates $\left(r^{2}\right)$. The SNP from the optimal multi-locus mixed-model (S2_157751802) was included as a covariate in the mixed linear model to control for the large-effect locus. None of the tested SNPs were significant at a 5\% FDR. 
we searched for more plausible candidate genes within $250 \mathrm{~kb}$ of other significant SNPs in LD with the peak SNP. This led to our primary focus on five SNPs significantly associated with cadmium that were in moderately strong LD (mean $r^{2}$ of 0.48 ) with the peak SNP and located within the heavy metal ATPase3 (hma3; Zm00001d005190) and heavy metal ATPase4 (hma4; Zm00001d005189) genes (Supplementary Table S5). Notably, the hma3 and hma4 genes, which were $\sim 630 \mathrm{~kb}$ from the peak SNP, encode proteins with 71 and $66 \%$ amino acid sequence identity to OsHMA3 (Supplementary Table S5), a $\mathrm{P}_{1 \mathrm{~B}}$-type ATPase involved in sequestration of cadmium in root vacuoles of rice (Ueno et al. 2010).

To better resolve the expansive association signal resulting from a large-effect locus located in a long range, high LD genomic region, a chromosome-wide multi-locus mixed-model procedure (MLMM) was conducted for cadmium. The resulting optimal model only included the peak SNP (S2_157751802) on chromosome 2 (Supplementary Table S6). When GWAS was reconducted with this MLMM-selected SNP included as a covariate in the mixed linear model to control for this large-effect locus on chromosome 2, all other previously significant associations on chromosomes 2 and 8 were no longer significant at a genome-wide FDR of $5 \%$ (Figure 1B).

We identified 21 SNPs that spanned a 1.21-Mb region on chromosome 7 that were significantly associated with the concentration of zinc in fresh kernels (Figure 2A). The peak SNP (S7_174515604; P-value $3.19 \times 10^{-10} ; 180,076,727 \mathrm{bp}$ ) for this association signal explained $7 \%$ of the phenotypic variance for zinc and was located within the ORF of a gene (Zm00001d022563) that encodes a tetratricopeptide repeat-like superfamily protein (www.maizegdb.org). Notably, this peak SNP was located $\sim 111 \mathrm{~kb}$ from the nicotianamine synthase5 (nas5) gene (Zm00001d022557; Supplementary Table S5), which encodes a class II NAS that presumably contributes to the production of the metal chelator nicotianamine (Zhou et al. 2013). Of the 21 detected SNP-zinc associations, SNP S7_174279369, which was located $\sim 160 \mathrm{~kb}$ from nas5 and in moderately strong LD $\left(r^{2}=0.49\right)$ with the peak SNP for zinc, also had a near significant association (FDR-adjusted P-value 0.06) with the concentration of iron in fresh kernels. When using the chromosome-wide MLMM procedure to better clarify the association signal complex within the 1.21-Mb region on chromosome 7 for zinc, only the peak SNP S7_174515604 was included in the optimal model (Supplementary Table S6). With the MLMM-selected peak SNP as a covariate, a conditional mixed model analysis did not detect any SNPs significantly associated with zinc (Figure 2B).

Compared to cadmium and zinc, a relatively weaker association signal consisting of seven significant SNPs was identified for boron concentration on chromosome 1. Collectively, these seven SNPs comprised a 98.99-kb interval. The peak SNP (S1_189146031; P-value $5.01 \times 10^{-7} ; 191,327,920$ bp), which was located within the ORF of a gene (Zm00001d031473) encoding a protein with $87 \%$ sequence identity to an aminoacylase in rice (Supplementary Table S5), explained 5\% of the phenotypic variance for boron concentration. Of the other candidate genes within $250 \mathrm{~kb}$ of the peak SNP position, a gene (Zm00001d031476) found to be $\sim 45 \mathrm{~kb}$ away from the peak SNP encodes a protein with 40-45\% amino acid sequence identity to two heavy metalassociated isoprenylated plant proteins (HIPPs) in Arabidopsis (Supplementary Table S5) that are putative metallochaperones (de Abreu-Neto et al. 2013). Indicative of a weaker effect locus, the

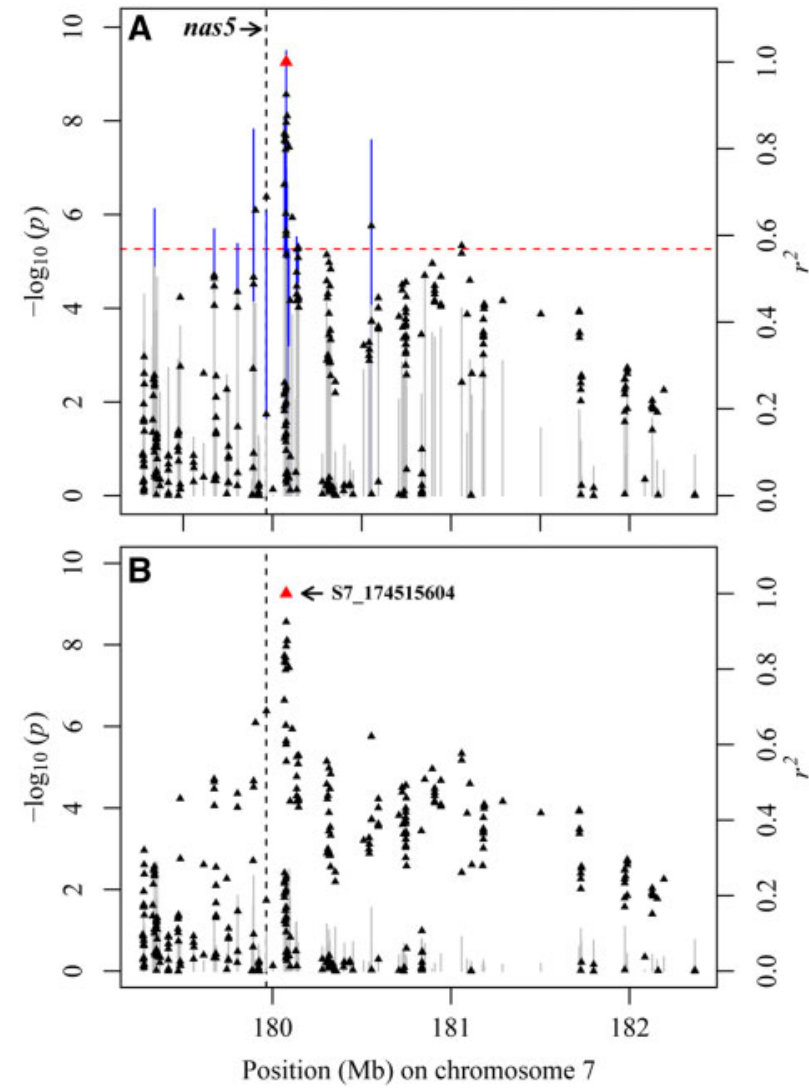

Figure 2 Genome-wide association study for zinc concentration in fresh kernels of sweet corn. (A) Scatter plot of association results from a mixed model analysis and linkage disequilibrium (LD) estimates $\left(r^{2}\right)$. The vertical lines are $-\log _{10} P$-values of single nucleotide polymorphisms (SNP), with the blue color representing SNPs that are statistically significant at a 5\% false discovery rate (FDR). Triangles are the $r^{2}$ values of each SNP relative to the peak SNP (indicated in red) at 180,076,727 bp (B73 RefGen_v4) on chromosome 7. The red horizontal dashed line indicates the $-\log _{10} P$-value of the least statistically significant SNP at a $5 \%$ FDR. The black vertical dashed line indicates the genomic position of the nicotianamine synthase5 (nas5; Zm00001d022557; 179,964,493-

$179,965,584 \mathrm{bp}$ ) gene. (B) Scatter plot of association results from a conditional mixed linear model analysis and LD estimates $\left(r^{2}\right)$. The SNP from the optimal multi-locus mixed-model (S7_174515604) was included as a covariate in the mixed linear model to control for the large-effect locus. None of the tested SNPs were significant at a 5\% FDR.

MLMM procedure at the chromosome-wide level did not select any SNP for the optimal model.

To identify marker-trait associations that may be locationspecific, we conducted GWAS for the 15 fresh kernel elemental phenotypes within each location (NY, Supplementary Figure S6; WI, Supplementary Figure S7), resulting in significant association signals detected for cadmium (NY and WI), zinc (NY and WI), nickel (NY), and calcium (WI) at 5\% FDR (Supplementary Table $\mathrm{S} 4)$. In the NY location, the association signal for cadmium consisted of 198 SNPs that defined a 36.89-Mb region on chromosome 2, with the peak SNP (S2_157751802; P-value $2.64 \times 10^{-19}$; $162,398,589 \mathrm{bp}$ ) for the signal the same as detected for cadmium across locations (Supplementary Table S4). Potentially the result of differential environmental variability combined with lower mapping precision from fewer evaluated inbred lines relative to the NY location (Supplementary Table S3), a different peak association signal (S2_159765450; P-value 1.98 $\times 10^{-15} ; 164,415,588 \mathrm{bp}$ ) that contained 81 SNPs covering 32.92-Mb on chromosome 2 was 
identified for cadmium in the WI location (Supplementary Table S4). The peak SNP for the WI location was $\sim 1.38-\mathrm{Mb}$ from hma3 and hma4, whereas the peak SNP in the NY location was $\sim 630 \mathrm{~kb}$ from the same two candidate genes. Within each location, the chromosome-level MLMM procedure was performed for cadmium (Supplementary Table S6), selecting only the peak SNP that when included as a covariate in a conditional GWAS rendered all other associations on chromosomes 1 (NY), 2 (NY and $\mathrm{WI}$ ), and 3 (NY and WI) no longer significant.

In both locations, the same SNP served as the peak association signal (NY: P-value $4.89 \times 10^{-8}$; WI: P-value $7.75 \times 10^{-8}$ ) for zinc, which was $\sim 111 \mathrm{~kb}$ from nas5 on chromosome 7 (Supplementary Table S4). The same SNP was also detected as the peak association signal for zinc across locations. The chromosome-wide MLMM approach selected only the peak SNP in the best model within each location (Supplementary Table S6). With the peak SNP as a covariate in a conditional GWAS, the other previously detected SNPs for zinc on chromosome 7 in NY (6 SNPs) and WI (3 SNPs) did not remain significant. Similar to the results from conducting the across-location GWAS, SNP S7_174279369, which was $\sim 160 \mathrm{~kb}$ from nas5, had a weak association (FDR-adjusted P-value 0.07 ) with iron in the NY location. In contrast to the across-location GWAS, however, this SNP was not significantly associated with zinc in either the NY or WI locations (Supplementary Table S4).

A significant association signal was detected for calcium in the WI location but not the NY location (Supplementary Table S4). This signal for calcium consisted of four SNPs on chromosome 10. The peak SNP (S10_124069084; P-value $6.09 \times 10^{-8}$; $125,112,781 \mathrm{bp}$ ) was $\sim 36 \mathrm{~kb}$ from a gene (Zm00001d025654) that codes for a protein with $47-49 \%$ sequence identity to two HIPPs in Arabidopsis (Supplementary Table S5). In addition, this peak SNP was selected by the MLMM in the optimal model at the chromosome-wide level (Supplementary Table S6). In concordance with other conditional GWAS results, no other SNPs remained significant when SNP S10_124069084 was used as a covariate in the mixed linear model.

Of the two field locations, we only detected a significant association for nickel in the NY location. The association signal for nickel on chromosome 9 consisted of four SNPs, with the peak SNP (S9_2213924; P-value $2.80 \times 10^{-8} ; 1,934,330 \mathrm{bp}$ ) for the signal selected by the MLMM in the optimal model (Supplementary Tables S4 and S6). The peak SNP was within the ORF of a gene (Zm00001d044768) that encodes a protein 39-47\% identical at the amino acid sequence level to three members of the NITRATE TRANSPORTER 1/PEPTIDE TRANSPORTER (NRT1/PTR) family (NPF) in Arabidopsis (Supplementary Table S5) that transport nitrate, amino acids, and hormones (Léran et al. 2014). In addition, this peak SNP was $\sim 44 \mathrm{~kb}$ from a gene (Zm00001d044771) coding a protein with $49-52 \%$ sequence identity to three matrix metalloproteinases (MMPs) in Arabidopsis (Supplementary Table S5) that have a zinc-binding sequence (Marino and Funk 2012). All other significant SNPs on chromosome 9 and a single significant SNP on chromosome 5 were no longer significantly associated with nickel in a conditional mixed linear model with the peak SNP as a covariate.

\section{Whole-genome prediction}

We evaluated the predictive ability of WGP with 147,762 SNP markers for the across-location concentrations of 15 elements that had been scored on fresh kernels of the 401 inbred lines. The 15 elements had an average predictive ability of 0.37 , ranging in abilities from 0.19 for rubidium to 0.52 for copper (Table 3 ). The predictive abilities were above average for iron (0.45) and zinc
Table 3 Predictive abilities (standard deviation) of whole-genome prediction models for 15 fresh kernel elemental phenotypes in the sweet corn association panel

\begin{tabular}{lcc}
\hline Trait & GBLUP & $\begin{array}{c}\text { GBLUP with endosperm } \\
\text { mutation type covariate }\end{array}$ \\
\hline Boron & $0.23(0.02)$ & $0.22(0.02)$ \\
Cadmium & $0.41(0.02)$ & $0.43(0.02)$ \\
Calcium & $0.27(0.03)$ & $0.27(0.03)$ \\
Copper & $0.52(0.01)$ & $0.53(0.01)$ \\
Iron & $0.45(0.02)$ & $0.45(0.02)$ \\
Magnesium & $0.40(0.02)$ & $0.40(0.02)$ \\
Manganese & $0.48(0.02)$ & $0.47(0.02)$ \\
Molybdenum & $0.25(0.03)$ & $0.25(0.03)$ \\
Nickel & $0.40(0.02)$ & $0.40(0.02)$ \\
Phosphorus & $0.47(0.02)$ & $0.47(0.02)$ \\
Potassium & $0.42(0.02)$ & $0.42(0.02)$ \\
Rubidium & $0.19(0.03)$ & $0.18(0.03)$ \\
Strontium & $0.32(0.02)$ & $0.32(0.02)$ \\
Sulfur & $0.26(0.02)$ & $0.27(0.02)$ \\
Zinc & $0.49(0.02)$ & $0.49(0.02)$ \\
Average & 0.37 & 0.37 \\
\hline
\end{tabular}

(0.49), suggesting that genomic selection could be used to increase the concentration of both nutritionally limiting microelements in fresh sweet corn kernels. A strong positive Pearson's correlation coefficient $(r=0.62 \mathrm{P}$-value $<0.05)$ was found between heritability estimates and predictive abilities for the 15 elemental phenotypes. Given the detection of significant differences among endosperm mutation types for 10 of the 15 elemental phenotypes (Table 2), endosperm mutation type was tested as an included covariate in WGP models, but changes to prediction abilities from its inclusion were zero to negligible (Table 3).

\section{Discussion}

Maintaining elemental homeostasis is critical for plants to realize optimal growth and complete their life cycle (Marschner 2011). In addition, the elemental content and composition of edible plant parts are influenced by genetic and environmental factors (Watanabe et al. 2007; Baxter and Dilkes 2012; Baxter et al. 2014). Several genes responsible for natural variation of elemental levels in root and shoot tissues have been identified and characterized in plants (Huang and Salt 2016; Yang et al. 2018), but considerable effort remains to pinpoint the genes regulating elemental levels in seed of crops. To further this research, we examined the extent of phenotypic variation for elemental concentrations in fresh kernels and performed a GWAS to identify candidate genes controlling this phenotypic variability in a sweet corn association panel. We also evaluated the ability of genomewide markers to predict elemental concentrations, providing insights into the potential of genomic selection for optimizing the elemental profile of fresh kernels for human health and nutrition, especially iron and zinc. To the best of our knowledge, this work is the most extensive quantitative genetic analysis of elemental concentrations in fresh kernels of sweet corn.

The rank order of average concentrations for the measured elements in fresh kernels from the sweet corn association panel was highly concordant with that observed for the same elements in physiologically mature grain from the maize Ames panel of nonsweet corn tropical and temperate inbred lines (Wu et al. 2021) and the B73 (dent; Su1) x IL14H (sweet corn; su1) recombinant inbred line (RIL) family of the U.S. NAM panel (Baxter et al. 2014). Our sweet corn association panel showed a range of 13.66-28.11 and 
18.58-32.10 $\mu \mathrm{g} \mathrm{g}^{-1}$ on a dry weight basis for iron and zinc, respectively. However, these ranges were both lower and narrower than that found for iron (14.62-36.33 $\mu \mathrm{g} \mathrm{g}^{-1}$; 3.29 S.D.) and zinc (12.59$52.32 \mu \mathrm{g} \mathrm{g}^{-1} ; 4.36$ S.D.) in physiologically mature grain from the maize Ames panel (Wu et al. 2021). With consideration of losses from processing and bioavailability, the recommended target level iron and zinc grain concentrations established by HarvestPlus, which has a primary focus on developing nations where nutritional deficiencies are prevalent, are 60 and $38 \mu \mathrm{g} \mathrm{g}^{-1}$ dry weight, respectively, for developing biofortified maize based on achieving 30-40\% of estimated average requirements for adult women (nonpregnant, nonlactating) when consuming $400 \mathrm{~g} \mathrm{~d}^{-1}$ of whole maize grain (Bouis and Welch 2010). Although the maximum iron concentration observed in our sweet corn association panel (28.11 $\mu \mathrm{g} \mathrm{g}^{-1}$ dry weight) is twofold lower than the HarvestPlus breeding target, there are six inbred lines that have zinc concentrations ranging from 30.00 to $32.10 \mu \mathrm{g} \mathrm{g}^{-1}$ dry weight. As it relates to the consumption of fresh sweet corn when not a primary source of daily calories, the estimated maximum fresh kernel concentrations observed in our association panel would provide approximately $4-9 \%$ and $7-10 \%$ of the RDA of iron and zinc, respectively, for adult nonelderly women (nonpregnant, nonlactating) and men when consuming $100 \mathrm{~g}$ ( 75\% water) of uncooked fresh sweet corn. Irrespective of lacking experimental data from bioavailability assays, our comparison of phenotypic distributions to dietary guidelines implies that the top 5\% highest ranking lines for iron ( $\geq 22 \mu \mathrm{g} \mathrm{g}^{-1}$ dry weight) and zinc ( $\geq$ $28 \mu \mathrm{g} \mathrm{g}^{-1}$ dry weight) concentrations have promise for establishing a biofortification program for sweet corn.

We assessed whether the concentrations of elements differed significantly among endosperm mutation group types. Of the 15 elements, copper, iron, manganese, potassium, and sulfur were highly significantly different $(P<0.001)$ between two or more endosperm mutation type groups (su1, sh2, and su1sh2) (Table 2). Relatedly, Baxter et al. (2014) showed that the content for four (iron, manganese, potassium, and sulfur) of these five elements significantly differed $(P<0.0005)$ between visibly "wrinkled" (su1/ su1) and "nonwrinkled" (Su1/su1 or Su1/Su1) kernels harvested at physiological maturity. As it relates to the spatial distribution of elements in sweet corn kernels, Cheah et al. (2019) analyzed immature (21 DAP) kernels from a single sweet corn (sh2) variety via synchrotron-based X-ray fluorescence microscopy to reveal that potassium and calcium were generally present throughout the kernel, sulfur concentrated mainly in the axis of the embryo and the periphery of the endosperm, and the scutellum of the embryo had at least 20-fold higher concentrations of phosphorus, iron, zinc, and manganese than in the endosperm. Notably, Cheah et al. (2019) also showed that these spatial distribution maps for elements were highly similar to those of immature maize (nonsweet corn) kernels. Despite these valuable insights from earlier studies, further experimental work will be needed to determine whether the observed significant difference in concentrations of the five elements among endosperm mutation type groups in our sweet corn association panel was attributed to variation in physiological, genetic, and/or physical attributes of fresh kernels.

Conducting GWAS across locations for the concentrations of elements in fresh kernels of the sweet corn association panel resulted in the identification of candidate genes associated with cadmium and zinc at the genome-wide level. Of these elements, the strongest association signal was for cadmium, having an association signal on chromosome 2 that spread more than $35-\mathrm{Mb}$ across a recombinationally inert genomic region. In addition, the peak SNP of this association signal co-localized with the single across-location QTL detected for grain cadmium concentration in the maize NAM panel (Supplementary Tables S4 and S7), but the GWAS resolution for this region in the NAM panel (Supplementary Table S8) was too limiting to convincingly identify an underlying causal gene (Ziegler et al. 2017). In our sweet corn association panel, however, two likely candidate causal genes were identified, hma3 and hma4, both having SNPs in moderately strong LD with the peak SNP of the association signal. These genes are two of a 12-member gene family encoding HMAs in the genome of maize inbred line B73 (Cao et al. 2019).

Of the 12 HMA genes, the proteins encoded by hma3 and hma4 have high sequence identity (Supplementary Table S5) to the $\mathrm{P}_{1 \mathrm{~B}}$-type ATPase, OsHMA3-a tonoplast-localized zinc/cadmium transporter that has been shown to be expressed in rice roots, mediates cadmium and zinc vacuolar sequestration and, as such, participates in zinc homeostasis and root-to-shoot cadmium translocation (Ueno et al. 2010; Miyadate et al. 2011; Sasaki et al. 2014; Cai et al. 2019). The loss-of-function of OsHMA3 has been associated with cadmium accumulation in rice grains, whereas lowcadmium rice cultivars express a functional OsHMA3 (Ueno et al. 2010). Furthermore, Ueno et al. (2010) have shown that the overexpression of OsHMA3 selectively decreased the accumulation of cadmium, but not other elements in the grain. In a follow-up study, Sasaki et al. (2014) showed that overexpression of OsHMA3 resulted in sequestration of both cadmium and zinc in rice root vacuoles, but the concentration of zinc in shoots was unaffected through the constitutive upregulation of transporter genes having putative involvement in the uptake and translocation of zinc. In agreement with Sasaki et al. (2014), we found no evidence of the large-effect locus spanning hma3 and hma4 having a significant association with zinc concentration in fresh sweet corn kernels. Importantly, Cao et al. (2019) identified several polymorphisms within hma3 to be significantly associated with leaf cadmium concentration at the seedling and adult plant stages in a maize diversity panel. Moreover, they further showed the expression level of hma3 to be highly upregulated in the roots of B73 in response to cadmium stress, whereas the expression of hma4 was undetectable in roots under the same conditions (Cao et al. 2019). Considering this, we propose that hma3 is the more likely of the two genes to have played a key genetic role in the accumulation of cadmium in fresh kernels.

The nas5 gene was found to be within $250 \mathrm{~kb}$ of the peak SNP for the across-location zinc and iron association signals on chromosome 7. These findings co-locate with the GWAS results of Ziegler et al. (2017) and Wu et al. (2021) from the U.S. maize NAM (Supplementary Tables S4, S7, and S8) and Ames mapping panels, respectively, that implicated nas 5 as a possible pleiotropic controller for the concentrations of both zinc and iron in physiologically mature grain samples. As one of nine gene family members in the B73 reference genome, nas5 phylogenetically groups together with nas3 and nas4, which together comprise class II nas genes (Zhou et al. 2013). The NAS enzyme encoded by nas5 is hypothesized to be involved in the production of the nonproteinogenic amino acid, nicotianamine, an efficient chelator of transition metals including zinc and iron (Takahashi et al. 2003; Curie et al. 2009; Swamy et al. 2016). In addition, to a suggested role in intracellular metal homeostasis, nicotianamine facilitates phloem-based metal delivery from source (e.g., leaves) to sink (e.g., seeds) tissues (Takahashi et al. 2003; Curie et al. 2009; Swamy et al. 2016). Nicotianamine also serves as a precursor for the synthesis of root-exuded mugineic acid-type phytosiderophores that chelate divalent metals for eventual root uptake (Curie et al. 2009; Swamy et al. 2016). 
Consistent with a proposed role of nas5 in long-distance metal transport rather than uptake into roots, Zhou et al. (2013) showed that transcripts of class II nas genes including nas5 accumulated mainly in maize leaves and sheath, whereas class I nas genes were predominantly expressed in maize roots. Interestingly, of the three class II nas genes, nas5 was more highly expressed in maize stems, further suggesting its contribution to long-distance metal transport and perhaps its contribution to metal loading to seeds (Zhou et al. 2013). In addition, the transcriptional expression level of nas5 was downregulated by iron deficiency in both shoots and roots but upregulated under excess iron and zinc in roots. Activation tagging of OsNAS3, the closest rice homolog of nas5 (Zhou et al. 2013), produced an increased level of nicotianamine that resulted in elevated levels of zinc and iron in shoots, roots, and seeds of activation-tagged rice plants (Lee et al. 2009). Despite the lack of functional validation, results from the maize association mapping and transgenic rice studies strongly support the nomination of nas5 as a causal gene for controlling zinc and iron concentrations in fresh sweet corn kernels.

Similar to our findings with nas5 in the sweet corn association panel, Wu et al. (2021) also identified a stronger association signal of nas5 with zinc relative to iron in the maize Ames panel. This finding is not entirely surprising considering that the affinity constants $(\mathrm{Kd})$ of complexes of nicotianamine with zinc is higher than for nicotianamine with iron (Curie et al. 2009; Gayomba et al. 2015). Therefore, it is plausible to propose that equimolar concentrations of iron and zinc would result in the selection of zinc for nicotianamine over iron (Curie et al. 2009; Gayomba et al. 2015). It is also noteworthy that the concentration of zinc in the phloem sap is thought to be higher than the concentration of iron (Reviewed in Gayomba et al. 2015), thus reinforcing the suggested role of nicotianamine and nas5 in zinc accumulation in fresh kernels.

In contrast to the highly probable causality of the hma3 and nas5 loci detected via GWAS across and within both locations, there is only moderately compelling evidence for the genetic involvement of identified candidate genes for the concentrations of boron (across locations), calcium (WI), and nickel (NY). Of the genes within $250 \mathrm{~kb}$ of the peak SNP for the boron association signal on chromosome 1, two genes encoding a putative protein with sequence identity to either an aminoacylase (Zm00001d031473) or HIPP (Zm00001d031476) were the most plausible candidates. Under boron deficiency, aminoacylases (metalloenzymes involved in amino acid metabolism) have been shown to have decreased protein and increased transcript levels in Brassica napus L. roots and Citrus sinensis leaves, respectively (Wang et al. 2010; Lu et al. 2015). Transcriptome profiling revealed a HIPP to be upregulated in leaves and roots of black poplar (Populus nigra L.) grown under boron toxicity (Yıldınm and Uylaş, 2016), which perhaps is not surprising given that HIPPs are metallochaperones involved in the transport of metallic ions and response to abiotic stresses (de Abreu-Neto et al. 2013). Despite these findings in other plant systems, the exact mechanism by which the identified aminoacylase and HIPP would have contributed to the accumulation of boron in fresh kernels is unknown but nevertheless merits further experimental investigation.

Although the peak SNP associated with calcium concentration in the WI location resided within a genomic region that lacked a definitive candidate gene, it was located $\sim 36 \mathrm{~kb}$ from a gene (Zm00001d025654) encoding a putative HIPP. However, to our knowledge, HIPPs have never been experimentally shown to bind $\mathrm{Ca}^{2+}$ (de Abreu-Neto et al. 2013), thus an in vitro study would be needed to determine whether $\mathrm{Ca}^{2+}$ is bound by the putative HIPP that Zm00001d025654 encodes. It is interesting that collectively, two different HIPP candidate genes were identified for the concentrations of boron and calcium, but these proteins would not be expected to have similar roles given that calcium and boron have different chemical and physiological properties (Marschner 2011). Regardless, these findings open new avenues of inquiry that could deepen our understanding of the genetic basis of boron and calcium accumulation in fresh sweet corn kernels.

In contrast to calcium, the nickel association signal for the NY location coincided with association signals detected for nickel grain concentrations in the maize NAM (Supplementary Tables S4, S7, and S8) and Ames panels (Ziegler et al. 2017; Wu et al. 2021). This still genetically unresolved signal consisted of two possible candidate genes, an MMP (Zm00001d044771) and NPF member (Zm00001d044768). Even though MMPs could conceivably bind nickel in place of zinc (Cerdà-Costa and Gomis-Rüth 2014), their proteolytic activities to remodel the extracellular matrix (Marino and Funk 2012) lack a clear connection to nickel transport or accumulation. In addition, the putative NPF member encoded by Zm00001d044768 is hypothesized to transport nitrate given its high sequence identity to members of the NPF5 subfamily in Arabidopsis (Supplementary Table S5) (Niño-González et al. 2019), thus reducing but not completely eliminating the possibility that this yet-to-be characterized protein transports nickel or a substrate that binds nickel.

We showed that, on average, moderate predictive abilities were achieved through the application of WGP for the acrosslocation concentrations of the 15 elements in fresh sweet corn kernels. In addition, these predictive abilities were found to be strongly correlated with heritability estimates, which coheres with expectations (Combs and Bernardo 2013). In accordance with these results, Wu et al. (2021) analyzed 11 of these 15 elements in mature grain samples from the maize Ames association panel that had been evaluated in a single location across two years, finding that the moderate predictive abilities of the 11 elements from WGP had a strong correlation with their heritabilities. Also, the prediction abilities presented in Table 3 suggest that endosperm mutation type did not need to be considered as a covariate in WGP models to capture the genetic differences between the three groups at the genome-wide marker density employed in this study. Given that we observed significant $G \times E$ interaction for boron, copper, iron, magnesium, and rubidium, accounting for $G \times E$ in WGP models could result in slightly improved predictive abilities for these five elements. In support of this supposition, a multi-environment model incorporating $\mathrm{G} \times \mathrm{E}$ resulted in higher average prediction abilities for the concentration of zinc in kernels from a tropical maize inbred panel and a double haploid population compared to those from single-environment models (Mageto et al. 2020). Therefore, this should be an area of further exploration when conducting multi-environment genomic selection for elemental phenotypes whether at the immature or mature stages of kernel development.

The results from GWAS can be used to better inform how best to successfully implement genomic selection for the concentrations of elements in fresh sweet corn kernels. Apart from cadmium (18\%) and zinc (7\%), which each had a single locus explaining more than $5 \%$ of the phenotypic variance in the across-location GWAS, we observed relatively weaker association signals for boron, iron, and the other 11 elements in fresh kernels. Furthermore, we did not identify all of the strong association signals for the grain concentrations of boron, copper, manganese, molybdenum, nickel, and zinc that had been detected in the maize Ames panel (Wu et al. 2021). Compared to the Ames panel study of Wu et al. (2021) that had more than 2000 maize inbred 
lines, it is likely that the size of the sweet corn association panel in our study was too underpowered to identify these loci, whether because of their lower allele frequencies and/or weaker effects. Regardless, we posit that these elemental phenotypes are generally more polygenic than carotenoid and tocochromanol levels in fresh kernels of sweet corn that have a more oligogenic inheritance (Baseggio et al. 2019, 2020), thus making elemental phenotypes less tractable for genetic dissection in the sweet corn association panel. Therefore, genomic selection is more advisable than marker-assisted selection as a breeding approach for selecting for the concentration of elements in fresh kernels (Lorenz et al. 2011; Desta and Ortiz 2014). However, it is still worthwhile to assess the inclusion of large-effect loci as fixed effects such as those for cadmium and zinc in WGP models, as it could result in higher prediction abilities in specific sweet corn breeding populations (Bernardo 2014).

\section{Conclusions}

We used a sweet corn association panel to study the quantitative genetics of natural variation for the concentrations of 15 elements in fresh kernels. Through an across-location GWAS, we strongly implicated the candidate causal genes nas5 with iron/ zinc and hma3 with cadmium. Given that iron and zinc accumulation in fresh kernels have a partially shared genetic basis, the genetic correlation between these two phenotypes can be leveraged with multi-trait genomic selection approaches to possibly exceed the prediction accuracy of single-trait genomic selection (Jia and Jannink 2012) for simultaneous genetic gains in zinc and iron concentrations. Such efforts would help to address iron and zinc deficiencies of women, children, and older adults in the U.S. (Clark 2008) where sweet corn is highly consumed as a fresh vegetable. Importantly, the across- and within-location association signals at the nas5 and hma3 loci were specific to zinc/iron and cadmium, respectively. This suggests that genomic selection for lower cadmium accumulation to reduce possible toxicity should not influence zinc accumulation in the kernel. Even though $100 \mathrm{~g}$ of fresh sweet corn (medium-sized ear) with the maximum concentration of cadmium found in this panel is estimated to provide less than $2 \%$ of the provisional tolerable intake for this element in a day $(0.8 \mu \mathrm{g} / \mathrm{kg}$ bw/day) (JECFA 2011) when consumed by a $70 \mathrm{~kg}$ person, efforts should be dedicated toward developing haplotype tagging SNP markers at the nas5 and hma3 loci for breeding sweet corn that has lower cadmium but higher bioavailable zinc and iron, considering that sweet corn can be grown in regions with naturally elevated cadmium levels, or with low zinc and iron levels.

\section{Acknowledgments}

The authors are grateful to Jenna Hershberger for providing valuable feedback on an earlier version of the manuscript. They thank the current and past members of the Tracy and Gore labs for their efforts in pollination, harvest, and sample preparation. They also thank Lily Hislop and Patrick Flannery for sharing information from the Wisconsin field trials. Mention of trade names or commercial products in this publication is solely for the purpose of providing specific information and does not imply recommendation or endorsement by the USDA. The USDA is an equal opportunity provider and employer.

M.B., O.K.V., and M.A.G. co-wrote the manuscript; M.B. led the data analysis; G.Z. performed the ICP-MS analyses and elemental quantifications; N.K. and M.M. provided overall management of panel growth (planting, pollination, harvesting); M.B., M.M., and J.C. generated the marker datasets; I.B. oversaw the ICP-MS analyses and provided biological interpretation; C.R.B. and J.P.H. uplifted the NAM JL-GWAS results; D.W. integrated and provided biological interpretation of the NAM JL-GWAS results; O.K.V. provided biological interpretation of the candidate gene associations; W.F.T., M.M., and E.S.B. constructed the association panel; E.S.B., M.E.S., W.F.T., and M.A.G. conceived and designed the project; M.A.G. oversaw the data analysis, project management, design, and coordination.

\section{Funding}

This research was supported by the National Institute of Food and Agriculture (NIFA); the USDA Hatch under accession numbers 100397 (M.A.G.), 1010428 (M.A.G.), 1013637 (M.A.G.), 1013641 (M.A.G.), and 142 AAC6861 0726004 (W.F.T.), HarvestPlus (M.A.G.), the National Science Foundation (IOS-1546657 to C.R.B. and M.A.G.), USDA-NIFA grant numbers 2021-67013-33798 (O.K.V.) and 2018-67013-27418 (O.K.V.), Cornell University startup funds (M.A.G.), and the USDA-ARS (E.S.B. and I.B.).

Conflicts of interest: None declared.

\section{Literature cited}

Akdemir D, Okeke UG. 2015. EMMREML: Fitting Mixed Models with Known Covariance Structures. Vienna, Austria: $\mathrm{R}$ Core Development Team. https://CRAN.R-project.org/package= EMMREML (Accessed: 2021 June 7).

Baseggio M, Murray M, Magallanes-Lundback M, Kaczmar N, Chamness J, et al. 2019. Genome-wide association and genomic prediction models of tocochromanols in fresh sweet corn kernels. Plant Genome. 12:180038.

Baseggio M, Murray M, Magallanes-Lundback M, Kaczmar N, Chamness J, et al. 2020. Natural variation for carotenoids in fresh kernels is controlled by uncommon variants in sweet corn. Plant Genome. 13:e20008.

Baxter I. 2009. Ionomics: studying the social network of mineral nutrients. Curr Opin Plant Biol. 12:381-386.

Baxter I. 2015. Should we treat the ionome as a combination of individual elements, or should we be deriving novel combined traits? J Exp Bot. 66:2127-2131.

Baxter I, Dilkes BP. 2012. Elemental profiles reflect plant adaptations to the environment. Science. 336:1661-1663.

Baxter IR, Ziegler G, Lahner B, Mickelbart MV, Foley R, et al. 2014. Single-kernel ionomic profiles are highly heritable indicators of genetic and environmental influences on elemental accumulation in maize grain (Zea mays). PLoS One. 9:e87628.

Benjamini Y, Hochberg Y. 1995. Controlling the false discovery rate: a practical and powerful approach to multiple testing. J R Stat Soc B. 57:289-300.

Bernardo R. 2014. Genomewide selection when major genes are known. Crop Sci. 54:68-75.

Bhullar NK, Gruissem W. 2013. Nutritional enhancement of rice for human health: the contribution of biotechnology. Biotechnol Adv. 31:50-57.

Bouis HE, Welch RM. 2010. Biofortification-A sustainable agricultural strategy for reducing micronutrient malnutrition in the global south. Crop Sci. 50:S20-S32.

Box GEP, Cox DR. 1964. An analysis of transformations. J R Stat Soc B. $26: 211-243$ 
Bradbury PJ, Zhang Z, Kroon DE, Casstevens TM, Ramdoss Y, et al. 2007. TASSEL: software for association mapping of complex traits in diverse samples. Bioinformatics. 23:2633-2635.

Broadley M, Brown P, Cakmak I, Rengel Z, Zhao F. 2012. Function of Nutrients. In: H. Marschner, editor. Marschner's Mineral Nutrition of Higher Plants. 3rd ed. San Diego: Academic Press. p. 191-248.

Cai H, Huang S, Che J, Yamaji N, Ma JF. 2019. The tonoplast-localized transporter OsHMA3 plays an important role in maintaining Zn homeostasis in rice. J Exp Bot. 70:2717-2725.

Cao Y, Zhao X, Liu Y, Wang Y, Wu W, et al. 2019. Genome-wide identification of ZmHMAs and association of natural variation in ZmHMA2 and ZmHMA3 with leaf cadmium accumulation in maize. PeerJ. 7:e7877.

Cerdà-Costa N, Gomis-Rüth FX. 2014. Architecture and function of metallopeptidase catalytic domains. Protein Sci. 23:123-144.

Chan-Rodriguez D, Walker EL. 2018. Analysis of yellow striped mutants of Zea mays reveals novel loci contributing to iron deficiency chlorosis. Front Plant Sci. 9:157.

Cheah ZX, Kopittke PM, Harper SM, O'Hare TJ, Wang P, et al. 2019. In situ analyses of inorganic nutrient distribution in sweetcorn and maize kernels using synchrotron-based X-ray fluorescence microscopy. Ann Bot. 123:543-556.

Chen J, Chen Z. 2008. Extended Bayesian information criteria for model selection with large model spaces. Biometrika. 95:759-771.

Clark SF. 2008. Iron deficiency anemia. Nutr Clin Pract. 23:128-141.

Combs E, Bernardo R. 2013. Accuracy of genomewide selection for different traits with constant population size, heritability, and number of markers. Plant Genome. 6:1-7.

Curie C, Cassin G, Couch D, Divol F, Higuchi K, et al. 2009. Metal movement within the plant: contribution of nicotianamine and yellow stripe 1-like transporters. Ann Bot. 103:1-11.

Davies L, Gather U. 1993. The identification of multiple outliers. J Am Stat Assoc. 88:782-792.

de Abreu-Neto JB, Turchetto-Zolet AC, de Oliveira LFV, Zanettini MHB, Margis-Pinheiro M. 2013. Heavy metal-associated isoprenylated plant protein (HIPP): characterization of a family of proteins exclusive to plants. FEBS J. 280:1604-1616.

Desta ZA, Ortiz R. 2014. Genomic selection: genome-wide prediction in plant improvement. Trends Plant Sci. 19:592-601.

Elshire RJ, Glaubitz JC, Sun Q, Poland JA, Kawamoto K, et al. 2011. A robust, simple genotyping-by-sequencing (GBS) approach for high diversity species. PLoS One. 6:e19379.

Gayomba SR, Zhai Z, Jung H-I, Vatamaniuk OK. 2015. Local and systemic signaling of iron status and its interactions with homeostasis of other essential elements. Front Plant Sci. 6:716.

Gilmour AR, Gogel BJ, Cullis BR, Thompson R, Butler D, et al. 2009. ASReml User Guide Release 3.0. Hemel Hempstead, UK: VSN International Ltd.

Glaubitz JC, Casstevens TM, Lu F, Harriman J, Elshire RJ, et al. 2014. TASSEL-GBS: a high capacity genotyping by sequencing analysis pipeline. PLoS One. 9:e90346.

Gore MA, Chia J-M, Elshire RJ, Sun Q, Ersoz ES, et al. 2009. A first-generation haplotype map of maize. Science. 326:1115-1117.

Guo R, Dhliwayo T, Mageto EK, Palacios-Rojas N, Lee M, et al. 2020. Genomic prediction of kernel zinc concentration in multiple maize populations using genotyping-by-sequencing and repeat amplification sequencing markers. Front Plant Sci. 11:534.

Hawkesford M, Horst W, Kichey T, Lambers H, Schjoerring J, et al. 2012. Functions of macronutrients. In: H. Marschner, editor. Marschner's Mineral Nutrition of Higher Plants. San Diego: Academic Press. p. 135-189.
Hill WG, Weir BS. 1988. Variances and covariances of squared linkage disequilibria in finite populations. Theor Popul Biol. 33: 54-78.

Hindu V, Palacios-Rojas N, Babu R, Suwarno WB, Rashid Z, et al. 2018. Identification and validation of genomic regions influencing kernel zinc and iron in maize. Theor Appl Genet. 131:1443-1457.

Hirschi KD. 2020. Genetically modified plants: nutritious, sustainable, yet underrated. J Nutr. 150:2628-2634.

Holland JB, Nyquist WE, Cervantes-Martínez CT. 2003. Estimating and interpreting heritability for plant breeding: an update. Plant Breed. Rev. 22:9-112.

Huang X-Y, Salt DE. 2016. Plant ionomics: from elemental profiling to environmental adaptation. Mol Plant. 9:787-797.

Hung H-Y, Browne C, Guill K, Coles N, Eller M, et al. 2012. The relationship between parental genetic or phenotypic divergence and progeny variation in the maize nested association mapping population. Heredity. 108:490-499.

Institute of Medicine 2001. Dietary Reference Intakes for Vitamin A, Vitamin K, Arsenic, Boron, Chromium, Copper, Iodine, Iron, Manganese, Molybdenum, Nickel, Silicon, Vanadium, and Zinc. Washington, DC: The National Academies Press.

JECFA 2011. Evaluations of the joint FAO/WHO expert committee on food additives. 73th Report of the Joint FAO/WHO Expert Committee on Food Additive. WHO Technical Report Series 960.

Jia Y, Jannink J-L. 2012. Multiple-trait genomic selection methods increase genetic value prediction accuracy. Genetics. 192: 1513-1522.

Kenward MG, Roger JH. 1997. Small sample inference for fixed effects from restricted maximum likelihood. Biometrics. 53:983-997.

Khan MA, Castro-Guerrero N, Mendoza-Cozatl DG. 2014. Moving toward a precise nutrition: preferential loading of seeds with essential nutrients over non-essential toxic elements. Front Plant Sci. 5:51.

Kurtz S. 2003. The Vmatch Large Scale Sequence Analysis Software-A Manual. http://www.vmatch.de/virtman.pdf (Accessed: 2021 June 7).

Lee S, Jeon US, Lee SJ, Kim Y-K, Persson DP, et al. 2009. Iron fortification of rice seeds through activation of the nicotianamine synthase gene. Proc Natl Acad Sci USA. 106:22014-22019.

Léran S, Varala K, Boyer J-C, Chiurazzi M, Crawford N, et al. 2014. A unified nomenclature of NITRATE TRANSPORTER 1/PEPTIDE TRANSPORTER family members in plants. Trends Plant Sci. 19: 5-9.

Li S, Liu X, Zhou X, Li Y, Yang W, et al. 2019. Improving zinc and iron accumulation in maize grains using the zinc and iron transporter ZmZIP5. Plant Cell Physiol. 60:2077-2085.

Linus Pauling Institute 2016. Micronutrient Information Center: Iron. https://pi.oregonstate.edu/mic/minerals/iron (Accessed: 2021 June 7).

Linus Pauling Institute 2019. Micronutrient Information Center: Zinc. https://lpi.oregonstate.edu/mic/minerals/zinc (Accessed: 2021 June 7).

Lipka AE, Gore MA, Magallanes-Lundback M, Mesberg A, Lin H, et al. 2013. Genome-wide association study and pathway-level analysis of tocochromanol levels in maize grain. G3 (Bethesda). 3: 1287-1299.

Lipka AE, Tian F, Wang Q Peiffer J, Li M, et al. 2012. GAPIT: genome association and prediction integrated tool. Bioinformatics. 28: 2397-2399.

Littell RC, Milliken GA, Stroup WW, Wolfinger RD, Schabenberger O. 2006. Appendix 1: linear mixed model theory. SAS for mixed models. Cary, NC: SAS Institute Inc. 
Lombi E, Scheckel KG, Pallon J, Carey AM, Zhu YG, et al. 2009. Speciation and distribution of arsenic and localization of nutrients in rice grains. New Phytol. 184:193-201.

Lombi E, Smith E, Hansen TH, Paterson D, de Jonge MD, et al. 2011. Megapixel imaging of (micro)nutrients in mature barley grains. J Exp Bot. 62:273-282.

Lönnerdal B. 2000. Dietary factors influencing zinc absorption. J Nutr. 130:1378S-1383S.

Lorenz AJ, Chao S, Asoro FG, Heffner EL, Hayashi T, et al. 2011. Genomic selection in plant breeding: knowledge and prospects. Adv Agron. 110:77-123.

Lu Y-B, Qi Y-P, Yang L-T, Lee J, Guo P, et al. 2015. Long-term boron-deficiency-responsive genes revealed by cDNA-AFLP differ between Citrus sinensis roots and leaves. Front Plant Sci. 6:585.

Lynch M, Walsh B. 1998. Genetics and Analysis of Quantitative Traits. Sunderland, MA: Sinauer.

Maathuis FJM. 2009. Physiological functions of mineral macronutrients. Curr Opin Plant Biol. 12:250-258.

Mageto EK, Crossa J, Pérez-Rodríguez P, Dhliwayo T, Palacios-Rojas N, et al. 2020. Genomic prediction with genotype by environment interaction analysis for kernel zinc concentration in tropical maize germplasm. G3 (Bethesda). 10:2629-2639.

Marino G, Funk C. 2012. Matrix metalloproteinases in plants: a brief overview. Physiol Plant. 145:196-202.

Marschner P. 2011. Marschner's Mineral Nutrition of Higher Plants. 3rd ed. Amsterdam, Netherlands: Elsevier/Academic Press.

Mengel K, Kirkby EA. 2001. Principles of Plant Nutrition. Dordrecht: Springer Science \& Business Media.

Miller JL. 2013. Iron deficiency anemia: a common and curable disease. Cold Spring Harb Perspect Med. 3:a011866.

Miyadate H, Adachi S, Hiraizumi A, Tezuka K, Nakazawa N, et al. 2011. OsHMA3, a P1B-type of ATPase affects root-to-shoot cadmium translocation in rice by mediating efflux into vacuoles. New Phytologist. 189:190-199.

Mocchegiani E, Romeo J, Malavolta M, Costarelli L, Giacconi R, et al. 2013. Zinc: dietary intake and impact of supplementation on immune function in elderly. Age (Dordr). 35:839-860.

Murgia I, Arosio P, Tarantino D, Soave C. 2012. Biofortification for combating "hidden hunger" for iron. Trends Plant Sci. 17:47-55.

Neter J, Kutner MH, Nachtsheim CJ, Wasserman W. 1996. Applied Linear Statistical Models. Chicago: Irwin.

Niño-González M, Novo-Uzal E, Richardson DN, Barros PM, Duque P. 2019. More transporters, more substrates: the Arabidopsis major facilitator superfamily revisited. Mol Plant. 12:1182-1202.

Pongrac P, Vogel-Mikuš K, Jeromel L, Vavpetič P, Pelicon P, et al. 2013. Spatially resolved distributions of the mineral elements in the grain of tartary buckwheat (Fagopyrum tataricum). Food Res Int. 54:125-131.

Prasad AS. 2014. Impact of the discovery of human zinc deficiency on health. J Trace Elem Med Biol. 28:357-363.

Core Team R. 2019. R: A Language and Environment for Statistical Computing. Vienna, Austria: R Foundation for Statistical Computing. https://www.R-project.org/ (Accessed: 2021 June 7).

Ricci WA, Lu Z, Ji L, Marand AP, Ethridge CL, et al. 2019. Widespread long-range cis-regulatory elements in the maize genome. Nat Plants. 5:1237-1249.

Rodgers-Melnick E, Vera DL, Bass HW, Buckler ES. 2016. Open chromatin reveals the functional maize genome. Proc Natl Acad Sci USA. 113:E3177-E3184.

Romay MC, Millard MJ, Glaubitz JC, Peiffer JA, Swarts KL, et al. 2013. Comprehensive genotyping of the USA national maize inbred seed bank. Genome Biol. 14:R55.
Sandstead HH, Smith JC. Jr, 1996. Deliberations and evaluations of approaches, endpoints and paradigms for determining zinc dietary recommendations. J Nutr. 126:2410S-2418S.

Sasaki A, Yamaji N, Ma JF. 2014. Overexpression of OsHMA3 enhances Cd tolerance and expression of Zn transporter genes in rice. J Exp Bot. 65:6013-6021.

Schroeder JI, Delhaize E, Frommer WB, Guerinot ML, Harrison MJ, et al. 2013. Using membrane transporters to improve crops for sustainable food production. Nature. 497:60-66.

Schwarz G. 1978. Estimating the dimension of a model. Ann Stat. 6: 461-464.

Segura V, Vilhjálmsson BJ, Platt A, Korte A, Seren Ü, et al. 2012. An efficient multi-locus mixed-model approach for genome-wide association studies in structured populations. Nat Genet. 44: 825-830.

Singh S, Parihar P, Singh R, Singh VP, Prasad SM. 2016. Heavy metal tolerance in plants: role of transcriptomics, proteomics, metabolomics, and ionomics. Front Plant Sci. 6:1143.

Sun G, Zhu C, Kramer MH, Yang S-S, Song W, et al. 2010. Variation explained in mixed-model association mapping. Heredity (Edinb). 105:333-340.

Swamy BPM, Rahman MA, Inabangan-Asilo MA, Amparado A, Manito C, et al. 2016. Advances in breeding for high grain zinc in rice. Rice (N Y). 9:49.

Swarts K, Li H, Romero Navarro JA, An D, Romay MC, et al. 2014. Novel methods to optimize genotypic imputation for low-coverage, next-generation sequence data in crop plants. Plant Genome. 7:2014-2005.

Takahashi M, Terada Y, Nakai I, Nakanishi H, Yoshimura E, et al. 2003. Role of nicotianamine in the intracellular delivery of metals and plant reproductive development. Plant Cell. 15:1263-1280.

Ueno D, Yamaji N, Kono I, Huang CF, Ando T, et al. 2010. Gene limiting cadmium accumulation in rice. Proc Natl Acad Sci USA. 107: 16500-16505.

USDA-ARS 2019. FoodData Central. (Accessed 20214 Feb). https:// fdc.nal.usda.gov

USDA-NASS 2018. Vegetables 2017 summary. (Accessed 20214 Feb). https://usda.library.cornell.edu/concern/publications/ 02870v86p?locale=en.

VanRaden PM. 2008. Efficient methods to compute genomic predictions. J Dairy Sci. 91:4414-4423.

Viteri FE. 1998. A new concept in the control of iron deficiency: community-based preventive supplementation of at-risk groups by the weekly intake of iron supplements. Biomed Environ Sci. 11:46-60.

Von Wiren N, Mori S, Marschner H, Romheld V. 1994. Iron inefficiency in maize mutant ys1 (Zea mays L. cv Yellow-Stripe) is caused by a defect in uptake of iron phytosiderophores. Plant Physiol. 106:71-77.

Wang Z, Wang Z, Shi L, Wang L, Xu F. 2010. Proteomic alterations of Brassica napus root in response to boron deficiency. Plant Mol Biol. 74:265-278.

Watanabe T, Broadley MR, Jansen S, White PJ, Takada J, et al. 2007. Evolutionary control of leaf element composition in plants. New Phytol. 174:516-523.

Waters BM, Sankaran RP. 2011. Moving micronutrients from the soil to the seeds: genes and physiological processes from a biofortification perspective. Plant Sci. 180:562-574.

Whitt L, Ricachenevsky FK, Ziegler GZ, Clemens S, Walker E, et al. 2020. A curated list of genes that affect the plant ionome. Plant Direct. 4:e00272.

Wolfinger R, Federer WT, Cordero-Brana O. 1997. Recovering information in augmented designs, using SAS PROC GLM and PROC mixed. Agronj. 89:856-859. 
Wu D, Tanaka R, Li X, Ramstein GP, Cu S, et al. 2021. High-resolution genome-wide association study pinpoints metal transporter and chelator genes involved in the genetic control of element levels in maize grain. G3 (Bethesda). 11:jkab059.

Yang M, Lu K, Zhao F-J, Xie W, Ramakrishna P, et al. 2018. Genome-wide association studies reveal the genetic basis of ionomic variation in rice. Plant Cell. 30:2720-2740.

Ylldınm K, Uylaş S. 2016. Genome-wide transcriptome profiling of black poplar (Populus nigra L.) under boron toxicity revealed candidate genes responsible in boron uptake, transport and detoxification. Plant Physiol Biochem. 109:146-155.

Yu J, Pressoir G, Briggs WH, Vroh Bi I, Yamasaki M, et al. 2006. A unified mixed-model method for association mapping that accounts for multiple levels of relatedness. Nat Genet. 38:203-208.

Zang J, Huo Y, Liu J, Zhang H, Liu J, et al. 2020. Maize YSL2 is required for iron distribution and development in kernels. J Exp Bot. 71: 5896-5910.
Zhang Z, Ersoz E, Lai C-Q, Todhunter RJ, Tiwari HK, et al. 2010. Mixed linear model approach adapted for genome-wide association studies. Nat Genet. 42:355-360.

Zhou X, Li S, Zhao Q, Liu X, Zhang S, et al. 2013. Genome-wide identification, classification and expression profiling of nicotianamine synthase (NAS) gene family in maize. BMC Genomics. 14:238.

Zhou X, Stephens M. 2014. Efficient multivariate linear mixed model algorithms for genome-wide association studies. Nat Methods. 11:407-409.

Ziegler G, Kear PJ, Wu D, Ziyomo C, Lipka AE, et al. 2017. Elemental accumulation in kemels of the maize nested association mapping panel reveals signals of gene by environment interactions. BioRxiv 164962.

Ziegler G, Terauchi A, Becker A, Armstrong P, Hudson K, et al. 2013. Ionomic screening of field-grown soybean identifies mutants with altered seed elemental composition. Plant Genome. 6:2012-2007.

Communicating editor: R. Dawe 